\title{
Tecnología y bienestar
}

\section{Technology and well-being}

\author{
Javier Castellanos ${ }^{1 \mathrm{a}}$, René Carreño ${ }^{1 \mathrm{~b}}$, Werner Hofacker ${ }^{2}$ \\ ${ }^{1}$ Universidad Pontificia Bolivariana, Seccional Bucaramanga, Colombia. Correo electrónico: a javier.castellanos@upb.edu.co, \\ b rene.carreno@upb.edu.co \\ ${ }^{2}$ Hochschule Kontanz University of Applied Sciences, Germany. Correo electrónico: hofacker@htwg-konstanz.de
}

Recibido: 21 septiembre, 2017. Aceptado: 23 agosto, 2018. Versión final: 24 octubre, 2018.

\begin{abstract}
Resumen
La tecnología es responsable de la transformación del hombre y de la humanidad; es una respuesta innata al empeño de los individuos por vivir en el mundo y "estar bien". No obstante, el crecimiento económico de las naciones entendido por muchos como el mejoramiento del bienestar de sus ciudadanos y el desarrollo tecnológico- presenta una relación fuerte con el deterioro del medioambiente y las consecuencias adversas que podría acarrear para todo el planeta. Teniendo en cuenta lo anterior, se podría suponer que si una comunidad incrementa su bienestar su hábitat podría verse seriamente deteriorado, lo cual establece una clara contradicción entre "bienestar" y la calidad del "estar", esta última definida como la existencia física del ser humano en el hoy y en el ahora. Por todo lo anterior, este trabajo pretende identificar qué tipo de relación tienen la innovación tecnológica y el bienestar humano. Para ello, se reflexionó acerca de la necesidad de "bienestar" inherente del hombre, la innovación tecnológica ligada a este estado de bienestar y sus consecuencias en el "estar" mismo. Se concluye que existe una relación muy estrecha, ya que la innovación tecnológica hace parte de la esencia de la raza humana; sin embargo, mientras el desarrollo de la tecnología esté orientado por intereses particulares, ligados a poderes económicos y políticos, la relación entre "bienestar" y la calidad del "estar" tendrán una relación inversa. La evolución del concepto de "bienestar" definido por Marcuse requiere el cambio del estilo de vida, y de esta forma la innovación debería estar enfocada en el diseño de tecnología menos nociva -amigable con el medioambiente-, que les permita a todos vivir bien, que no simplemente vivir mejor.
\end{abstract}

Palabras clave: desarrollo tecnológico; impacto ambiental; calidad de vida.

\begin{abstract}
Technology is responsible for man and humanity transformation; it is a natural response to human beings' efforts to live in this world and "being well". Nevertheless, nations' economic growth -understood by many as the improvement of their citizens well-being together with technological development-, shows a strong relation with environmental degradation and its adverse consequences worldwide. In view of the above, this work looks forward to identify what kind of relation has technological innovation with human well-being. To do this, it was conducted a careful reasoning on man's inherent need for "well-being", technological innovation tied to this well-being and its consequences to the "being" itself. It is concluded that there is a very close relation because technological innovation is part of the very essence of the human race; however, as long as technology development is oriented by particular interests tied to economic and political power, the relationship between "well-being " and the quality of "being" will be inversely related. The evolution of the concept of "well-being" as defined by Marcuse requires a change of lifestyle, and thus innovation should be focused on the design of less harmful technology -environmentally friendly-, allowing people to live well and not just to live better.
\end{abstract}

Keywords: technological development; environmental impact; quality of live.

ISSN impreso: 1657 - 4583, ISSN en línea: 2145 - 8456, CC BY-ND 4.0 (c) (i) $€$

Como citar: J. Castellanos, R. Carreño, W. Hofacker, "Tecnología y bienestar," Rev. UIS Ing., vol. 18, no. 1, pp. 237-242, 2019. doi: 10.18273/revuin.v18n1-2019021 


\section{Introducción}

El empeño del hombre por vivir, por estar en el mundo, es inseparable de su empeño por "estar bien" [1], lo que permite suponer que el bienestar es una búsqueda constante del hombre. Según Mumford [2], los artefactos tecnológicos son resultado del acto natural, reflexivo y consciente del hombre por mejorar su vida; sin embargo estos artefactos han disminuido su destreza para suplir muchas necesidades cuando no se poseen, y pareciera que al hombre contemporáneo le es imposible deshacerse de ellos.

Podría decirse que las comunidades actuales tienen la plena convicción de que los artefactos ofrecen el bienestar que requieren y reclaman. Esta convicción, junto con la ilusión de superioridad que acompaña al hombre, es la que favorece el desperdicio de los recursos [3]. Paradójicamente, hoy se realiza más esfuerzo que antes cuando se emprenden las mismas actividades; por ejemplo: aunque actualmente los sistemas de transporte se mueven más rápido, se debe recorrer mayores distancias para dirigirse del hogar al trabajo. Esto lleva a invertir casi el mismo tiempo que tomaba años atrás, pero con un gasto mayor de energía y un incremento inexorable de la polución de nuestro entorno.

Así mismo, de acuerdo con Ortega y Gasset [1], a diferencia de los animales, las necesidades del hombre, más que orgánicas, son superfluas. Para el hombre existe una necesidad cuando esta corresponde con sus condiciones de bienestar, y, por tanto, la tecnología podría considerarse como la producción de lo superfluo.

Pese a esto, el actual sistema socioeconómico predominante no permite develar otra alternativa, ya que el hombre no es capaz de percibir una vida sin tecnología. Según Habermas [4], el capitalismo está atado a los intereses de una economía privada, lo que lleva a un crecimiento económico continuo, autorregulado y a largo plazo. Esta visión permite suponer que los recursos naturales disponibles son infinitos e inagotables; sin embargo, esta premisa no es cierta.

Debido a su desmesurado crecimiento industrial, China se ha convertido recientemente en una gran potencia económica; su desarrollo ha permitido sacar de la pobreza a 300 millones de personas, pero esto ha supuesto consecuencias ecológicas altas para el país y el planeta [5]. En el año 2009, este país asiático volcaba al medioambiente más $\mathrm{CO}_{2}$ que Estados Unidos y Canadá juntos, con un aumento en sus emisiones desde el año 2000 del orden de $171 \%$ [6]. Con esta misma orientación, si bien está bien entendido que la mayoría de la reservas de combustibles fósiles deben permanecer bajo tierra para evitar un desastre ambiental de proporciones épicas, bajo la lógica del capitalismo, los propietarios de estas reservas compiten en la actualidad para explotarlas al máximo, enmarcadas, según ellos, en una doctrina de racionalidad [7].

De esta forma, se puede suponer que si un pueblo mejora su bienestar el estado de su hábitat se ve gravemente deteriorado; es decir, el "bienestar" tiene una relación inversa con la calidad del "estar", lo cual no es lógico. Pareciera que el planeta se ha convertido solo en un proveedor de materias primas que brindan el "estar bien" en detrimento del mismo "estar".

Así, en el marco de este trabajo, se pretende establecer qué tipo de relación tiene la innovación tecnológica con el bienestar humano. Para responder a ese interrogante se reflexionó acerca de la necesidad inherente del hombre de "estar bien", acerca de la innovación tecnológica atada a este estado de bienestar y sus consecuencias en el "estar" mismo.

\section{Reflexión}

\subsection{La búsqueda del estar bien}

El hombre, a diferencia de los animales, vive porque quiere hacerlo, y la búsqueda de entorno más confortable es una necesidad propia de su ser. Con el transcurso del tiempo, la adaptación del hombre al medio dio paso a una modificación del medio por el accionar del hombre, con un único propósito: el bienestar [1].

Los dispositivos mecánicos no son solo una ventaja que posee el ser humano para hacer, sino un factor cultural, histórico y transformador que ha cambiado la faz de la tierra. Esta capacidad creadora del hombre está enmarcada no solo en la fabricación, sino en la investigación e invención de objetos que le permitan mitigar la hostilidad del medioambiente. Pareciera que para el hombre lo real es defectuoso, y busca, por los medios que sean necesarios, amoldar un entorno para reducir la dureza con lo que lo trata [8]. Desde este punto de vista, pensar en lo que nos rodea como "no-amigo" es sugerir que la naturaleza es algo externo al hombre, es pensar entonces que el hombre no hace parte de la naturaleza.

En consecuencia, la necesidad de estar bien nace de una lucha por vivir y perdurar en un estado de bienestar, para lo cual se utiliza la tecnología, que se constituye a su vez en la responsable de la transformación del hombre y de la humanidad [8]. La tecnología se convierte, desde los inicios del hombre, en el motor que impulsa su capacidad creadora para el diseño de herramientas, objetos o ingenios mecánicos. 


\subsection{El significado de estar bien}

Hasta ahora se ha podido determinar que la noción de bienestar es necesaria para la vida como hombre y que es la responsable de potenciar su capacidad creadora para el diseño de herramientas, objetos o aparatos mecánicos, pero la noción de bienestar no es estática y cambia a través del tiempo.

Las necesidades del hombre son, en su contenido, manifestación concreta de necesidades históricas condicionadas por la evolución histórica del mismo hombre [9]. Por tanto, es posible hilvanar las necesidades humanas a partir del análisis de la razón de uso de los objetos en la historia.

Según Llovet [10], las sociedades primitivas lograron descubrir los fenómenos de la naturaleza, lo que les permitió crear por analogía una cultura llena de objetos. En esta fase naturista, los diseños eran resultado de una casualidad, más que de una reflexión. Los objetos constituían la solución más adecuada y la única posible a un problema concreto, es decir, en estas sociedades no existían objetos inútiles que fueran construidos para que no cumplieran ninguna función.

Cuando el análisis acerca de las soluciones empezó a hacer parte de una memoria histórica, se dio origen a la fase inventiva en el desarrollo de objetos. Los objetos en esta etapa no nacen de la articulación de elementos naturales, sino de elementos culturales que obedecieron a un trabajo previo y reflexivo en el seno de lo natural, y a una transformación de lo natural en un objeto; es una cultura con memoria y consciente de lo que hace. La necesidad misma no es importante, lo importante son las necesidades que se crean en función del marco heredado de la cultura [10].

Por último, a partir de la revolución industrial, toda relación de elaboración que el hombre tiene con el objeto se pierde debido a la producción en masa. El consumismo establece como imperativo el deseo de poseer más y más objetos que muchas veces son innecesarios. En esta fase consumista, los objetos están dotados de poca o ninguna utilidad, además de que tienen incorporados un peculiar valor de cambio-signo [10]. Esto lleva a poseer tecnología inducida por las necesidades que la sociedad demanda [10], [11].

\subsection{La influencia de la interconexión social}

De acuerdo con el pensamiento aristotélico, las decisiones sociales del hombre parten de un proceso reflexivo en donde su elección depende de otros y en donde las circunstancias limitan el marco de acción de lo posible. Camps [12] señala que el desarrollo tecnológico modificó este proceso de decisión social, puesto que desde su aparición lo imposible y lo necesario pasan a hacer parte del marco de lo posible, y ya no existen límites claros. Debido a esta libertad, el ser humano cree que las decisiones sociales no dependen de otros, y la responsabilidad se alude bajo el criterio del interés común consensuado. El hecho de que el hombre tome sus propias decisiones bajo un interés común definido por consenso podría suponer que lo decidido es conveniente para todos. Sin embargo, Marcuse [11] señala que la aparente libertad que poseemos está condicionada a intereses creados por terceros, que permiten la manipulación de la sociedad. Se convierten entonces en particulares y no comunes. Estos intereses particulares promueven la política de despilfarro, para producir y consumir desproporcionadamente. Se establece entonces una forma de libertad engañosa, en la que se da al hombre a escoger lo que ya ha sido escogido por otros. De acuerdo con Chomsky [7], los bienes en poder de particulares, no aquellos que hacen parte del patrimonio común, se enfrentan a su destrucción, por el deseo de enriquecimento de los primeros, que invevitablemente arrastrarán consigo a todos los demas.

\subsection{La innovación tecnológica y el bienestar}

La transformación debe ser para nuestro beneficio, teniendo en cuenta que el hombre siempre busca lo mejor; sin embargo, no siempre lo mejor es lo más benéfico.

Son muchos los ejemplos en la historia reciente de la humanidad que se pueden mencionar. De una parte, se sostiene que una de las ventajas más notables que justifica el uso de la energía nuclear en aplicaciones civiles tiene que ver con el hecho de que evita un amplio espectro de problemas que aparecen cuando se queman combustibles fósiles, particulamente el calentamiento global, las lluvias ácidas y la contaminación del aire. Además, se argumenta que los residuos tienen como ventaja importante que se pueden almacenar facilmente bajo tierra a grandes profundidades, en razón de las pequeñas cantidades de combustible atómico agotado que se producen [13]. Dando por cierto lo anterior, si ocurre un evento castastrófico, como el desencadenado por el terremoto y posterior tsunami que devastó el oriente de Japón en marzo de 2011, se pueden producir fallos que conduzcan a la fusión del núcleo del reactor y a la liberación de radiación al exterior. También puede escapar a la atmósfera, por accidente, el vapor de agua del circuito primario, que está contenido en el reactor y que es radioactivo. La radiación emitida puede alterar la información genética, provocar cáncer, malformación en los fetos, sin contar con el daño al ecosistema, del cual necesariamente dependemos. 
Por otra parte, los cultivos transgénicos desarrollados con el propósito fundamental de alimentar al hombre tienen un potencial enorme para resolver problemas de desnutrición y hambre que aquejan una porción importante de la población mundial. Son cultivos más resistentes a plagas, a las sequías, a las temperaturas extremas, a los herbicidas; crecen rápidamente y con mejor calidad. Sin embargo, aunque oficialmente no se conocen desventajas, algunos documentos elaborados por la Organización de las Naciones Unidas para la Agricultura y la Alimentación indican que su consumo podría originar una resistencia a los antibióticos, mayores residuos tóxicos en los alimentos, posibilidad de generación de nuevas alergias y riesgo de muerte de insectos polinizadores, con un gran impacto ecológico, entre otros [14], [15].

Los ejemplos muestran que a pesar de que existen desventajas inherentes al uso de ciertas tecnologías, que podrían por sí mismas amenazar la vida en este planeta, aún se utilizan abocando el fin con el cual fueron inventadas. Pese a su potencial dañino, el objeto no se modifica, incluso cuando el fin puede conseguirse con artefactos menos letales. El poder de estos objetos técnicos corrompe al hombre, pues no se utiliza para conseguir un mundo mejor, sino para dominar el mundo.

\subsection{La ingeniería, el motor del desarrollo tecnológico}

Es claro que la ingeniería fue concebida como una herramienta para la solución de problemas que afectan la actividad cotidiana de la sociedad. A pesar del hecho de que la formación del ingeniero se soporta en el manejo eficiente de los recursos y las fuerzas de la naturaleza en beneficio de esta sociedad, es gracias a la aplicación que los ingenieros hacen del avance en el conocimiento científico que surgen gran parte de las innovaciones responsables del deterioro de nuestro mundo y la explotación extensiva de los recursos naturales del planeta. Las soluciones tecnológicas de hoy se convierten por esta vía en los grandes problemas del futuro. La presión ejercida para lograr soluciones rápidas que resuelvan problemas puntuales de la sociedad no permite evaluar el impacto que los desarrollos tecnológicos producen en el entorno; impactos que, a largo plazo, producen problemas, como el efecto invernadero o desastres ecológicos por accidentes industriales. Surgen en consecuencia varios interrogantes: ¿va la ingeniería en la dirección correcta? ¿Se están arraigando los valores correctos en los ingenieros? ¿Qué criterios se están usando para medir el impacto de los desarrollos ingenieriles y los productos?

\section{Discusión}

Es difícil percibir que antes de nuestra época las intervenciones del hombre en la naturaleza, tal y como él mismo las veía, eran esencialmente superficiales e incapaces de dañar su permanente equilibrio. La naturaleza no era objeto de la responsabilidad humana; ella se cuidaba a sí misma, pero actualmente sabemos que esto no es cierto y que es vulnerable.

A la luz de lo que ocurre hoy en el mundo, podría considerarse al hombre como un elemento hostil hacia la naturaleza, puesto que le ha ocasionado daños irreparables a corto plazo, situación que pone en riesgo no solo su supervivencia, sino la de todos los seres vivos que en ella habitan. El filósofo argentino Santiago Kovadloff resume esta reflexión de la siguiente forma: "Durante centenares de miles de años, el hombre luchó para abrirse un lugar en la naturaleza. Por primera vez en la historia de nuestra especie, la situación se ha invertido, y hoy es indispensable hacerle un lugar a la naturaleza en el mundo del hombre" [16].

El mismo Heidegger planteaba que la amenaza de que las máquinas actúen de forma mortífera no viene principalmente de ellas, sino de la preferencia del hombre por dominar, y que lamentablemente esta característica autoritaria limita la posibilidad de desocultar elementos más originarios y de experimentar otro tipo de verdad [17].

Por otra parte, la falsa noción de calidad de vida del mundo occidental favorece el despilfarro de recursos. Si bien para Aristóteles la felicidad del hombre no estaba condicionada a los bienes externos ni a la dominación del mar y la tierra, para la civilización actual existe una entera convicción de que son los aparatos mecánicos los que ofrecen la calidad de vida y que cuantos más objetos se posean, tanto mayor será la calidad de vida.

Se puede observar que el mundo es más rico que en épocas anteriores. El valor anual de la producción mundial de bienes de consumo y servicio ha crecido 20 veces más, en comparación con el siglo $\mathrm{XX}$, lo que significa que las personas tienen más objetos, aparatos mecánicos o herramientas en sus vidas [18]. Pero este éxito económico está haciendo estragos en nuestro hábitat; se están destruyendo los bosques, se aumentan los desiertos, se extinguen miles de especies vegetales y animales, se reduce la capa de ozono y se eleva la temperatura del planeta debido a la excesiva emisión de dióxido de carbono $\left(\mathrm{CO}_{2}\right)$. Todo debido al deseo delirante del poder económico y político. En el capitalismo, todos los actores económicos dependen de las exigencias de la competencia del mercado y la creciente productividad del trabajo para suplir sus 
necesidades básicas. De esta forma, el poder económico proporciona el poder político, en cuanto los obreros dependen de su trabajo para poder vivir [19].

\section{Conclusiones}

Con base en lo anterior, se concluye que existe una relación muy estrecha entre la innovación tecnológica y el estar bien, ya que la innovación tecnológica hace parte de la esencia misma de la raza humana; sin embargo, mientras el direccionamiento del desarrollo tecnológico esté atado a intereses particulares de poder económico y político, la relación entre "estar bien" y la calidad del "estar" tendrá una relación inversa.

Las alternativas de solución no deben estar orientadas al detrimento del desarrollo tecnológico, ya que actualmente no se puede vivir sin artefactos o aparatos mecánicos, pero tampoco se puede vivir en contra de la naturaleza. Es evidente que el progreso de la tecnología ha hecho al hombre cada vez más dependiente de ella, y por eso adquiere vital importancia el papel que juegan de los nuevos ingenieros, quienes proporcionan las soluciones que el mundo demanda, pero que también deben involucran en ellas un concepto más amplio de "innovación", donde se adopten permanentemente tecnologías amigables con el medioambiente, se creen dispositivos más eficientes y se haga un uso racional de los recursos disponibles. La formación actual de estos ingenieros requiere una visión holística del papel que desempeñan, a fin de arraigar en ellos la cultura de la eficiencia por encima de todo y sumergiéndolos en la cultura de la no violencia y la intervención amigable en la naturaleza.

\section{Referencias}

[1] J. Ortega y Gasset, Ensimismamiento y alteración; Meditación de la técnica y otros ensayos. Alianza Editorial, 2014.

[2] L. Mumford and C. Aznar de Acevedo, "Ventajas e inconvenientes," in Técnica y civilización, Madrid: Alianza Editorial, 1998, pp. 298-341.

[3] L. Mumford, Técnica y civilización. Madrid: Alianza Editorial, 1998.

[4] J. Habermas and M. Jiménez Redondo, Teoría de la acción comunicativa complementos y estudios previos. Cátedra, 1989.

[5] J. Farndon, China rises: how China's astonishing growth will change the world. Virgin Books, 2008.
[6] S. Rogers and L. Evans, "World carbon dioxide emissions data by country: China speeds ahead of the rest," The guardian, 2011. [En línea]. Disponible en:http://www.theguardian.com/news/datablog/2011/jan /31/world-carbon-dioxide-emissions-country-data-co 2 .

[7] N. Chomsky, "Magna Carta Messed Up the World, Here's How to Fix It," The nation, 2015. [En línea]. Disponible en: https://www.thenation.com/article/kill ing- commons/

[8] F. Dessauer, Discusión sobre la técnica. Rialp, 1964.

[9] L. García-Vega, J. Moya-Santoyo, and S. RodríguezDomínguez, Historia de la psicología. Siglo Veintiuno, 1992.

[10] J. Llovet, Ideología y metodología del diseño. Gustavo Gili, 1979.

[11] H. Marcuse, El hombre unidimensional: ensayo sobre la ideología de la sociedad industrial avanzada. Ed. Ariel, 2010.

[12] V. Camps, Una vida de calidad: reflexiones sobre bioética. Ares y Mares (Editorial Crítica), 2001.

[13] B. L. Cohen, The nuclear energy option: an alternative for the 90s. Plenum Press, 1990.

[14] Organiz.de las Nac. Unidas para la Agricult. y la Aliment., Los organismo modificados genéticamente, los consumidores, la incouidad de los alimentos y el medio ambiente. Organización de las Naciones Unidas para la Agricultura y la Alimentación, 1996.

[15] Organiz.de las Nac. Unidas para la Agricult. y la Aliment., El estado mundial de la agricultura y la alimentación, 2003-04. Organización de las Naciones Unidas para la Agricultura y la Alimentación, 2004.

[16] J. M. Alfaro Barbosa, G. Á. Martínez Turanzas, and G. T. Medina, Ambiente y sustentabilidad: por una educación ambiental. Universidad Autónoma de Nuevo León, 2014.

[17] M. Heidegger, F. Soler, J. Acevedo, and M. T. Poupin Oissel, Filosofía, ciencia y técnica. 2017.

[18] R. L. Sivard, A. T. Durning, and A. Braver, El planeta en la encrucijada: gastos militares, sociales y crisis ecológica. Icaria, 1992.

[19] E. M. Wood and D. González, El imperio del capital. Viejo Topo, 2004. 